\title{
Physiological considerations related to the pediatric airway
}

\author{
Ban C. H. Tsui, MD
}

Received: 8 January 2011 / Accepted: 19 January 2011/Published online: 10 February 2011

(C) Canadian Anesthesiologists' Society 2011

\section{To the Editor,}

Anatomical differences between adult and pediatric airways are well documented in the literature. ${ }^{1}$ Compared with adults, infants have a proportionately larger tongue situated entirely within the oropharynx; they are obligate nose breathers, and they have narrower nostrils in relation to the trachea, a higher and smaller larynx, and an elongated more rigid omega-shaped epiglottis. For all of these anatomical reasons, infants are susceptible to airway obstruction, respiratory failure, and difficult endotracheal intubation. The descent of the larynx in the first year of life is a characteristic exclusive to hominids - the larynx and epiglottis descend away from the soft palate creating a common passage for air, food, and liquid, and the base of the tongue becomes the anterior wall of the oropharynx.

Notable for its relative absence in the anesthesia literature is an explanation of these physiological airway changes. Of interest, however, is the following explanation put forward by anatomist, Dr. Crelin, over two decades ago. $^{2}$ The position of the infant's tongue entirely within the oral cavity allows the distinctly omega-shaped epiglottis to interlock with the soft palate when breastfeeding. This forms a barrier, creating a straight route for air to travel from nose to lungs while breast milk flows through the faucium channels, thus allowing the infant to breathe and swallow simultaneously (Figure). ${ }^{3}$ This characteristic is crucial for the infant's growth and well-being, as it

B. C. H. Tsui, MD ( $\square)$

University of Alberta, Edmonton, AB, Canada

e-mail: btsui@ualberta.ca optimizes her/his feeding ability. In addition to other developmental changes, the descent of the larynx during infancy and early childhood allows the wide range of sounds that enable effective communication. While vocal communication can be viewed as a survival-enhancing trait, the airway changes that enable it also result in a loss of the ability to breathe and drink simultaneously.

What are the clinical implications of these observations? Although we have no solid evidence to prove his interpretation of human airway changes, Crelin's research does provide us with a plausible analysis. His remarkable findings may explain why neonates and small infants display a preference for nasal breathing ("obligate nasal breather") ${ }^{1}$; why nasal flaring is one of the most important signs of airway obstruction in this population ${ }^{1}$; why nasal intermittent positive pressure ventilation via nasal prongs is possible and effective in neonates but not in adults ${ }^{4}$; why the highest incidence of dislodgements and delayed airway obstructions are seen when using a Laryngeal Mask Airway $\left(\mathrm{LMA}^{\mathrm{TM}}\right)$ in small infants; and why infants also have a higher incidence of the epiglottis impinging on the LMA. ${ }^{5}$ At the very least, Dr. Crelin's insights provide a method to help clinicians memorize these clinical observations. Crelin also theorized that the developmental descent of the epiglottis at four to six months of age represents a critical respiratory transition that could explain some cases of Sudden Infant Death Syndrome, which peaks at three to five months of age.

In the words of Einstein, "Look deep into nature, and then you will understand everything better". This article highlights the nature of human airway development from pediatrics to adults, providing further context in which to understand airway management and encourage the reader to seek the truth behind what we already appreciate regarding pediatric airway management. 
Figure The interlocking soft palate and epiglottis in infants due to the elevation of the larynx allows simultaneous breathing and drinking. (left) The position of the infant's tongue entirely within the oral cavity allows the distinctly omega-shaped epiglottis to interlock with the soft palate when feeding. (right) Milk flows through the lateral faucium channels. (Reproduced and adapted with permission from Principles of Airway Management $)^{3}$
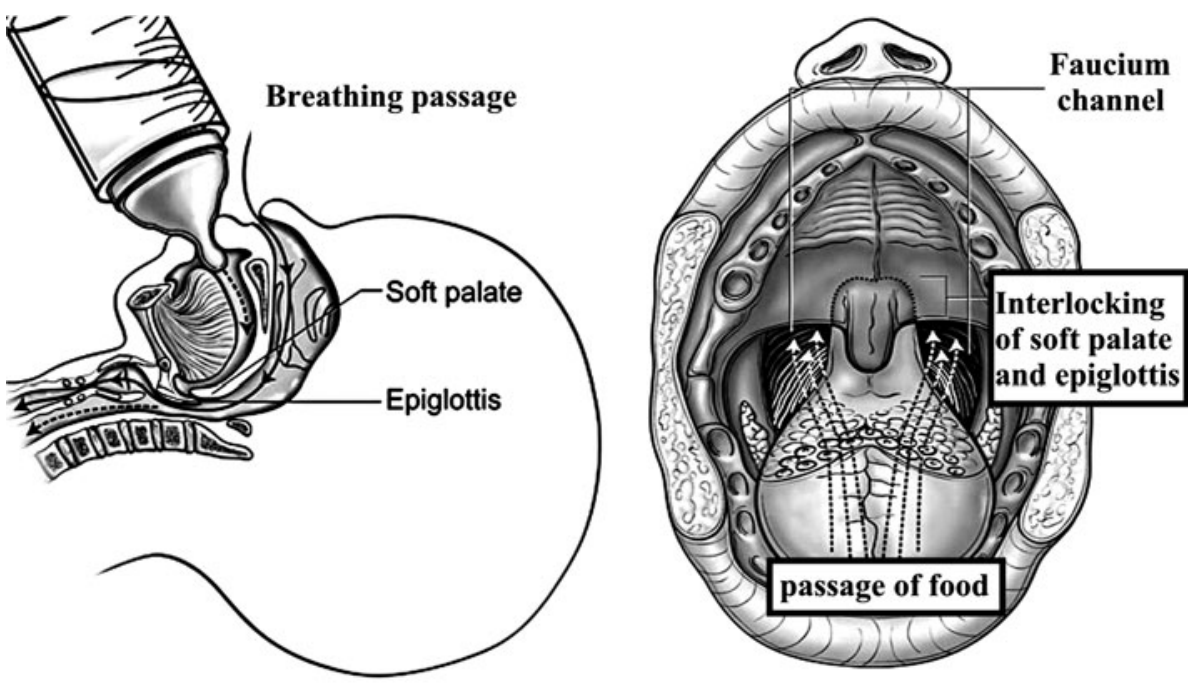

Simultaneous Breathing and Drinking
Competing interests None declared.

\section{References}

1. Adewale L. Anatomy and assessment of the pediatric airway. Paediatr Anaesth 2009; 19(Suppl 1): 1-8.

2. Crelin ES. The Human Vocal Tract: Anatomy, Function, Development, and Evolution. NY: Vantage Press; 1987.
3. Tsui BC. Pediatric airway management. In: Finucane BT, Tsui BC, Santora AH, editors. Principles of Airway Management. 4th ed. NY: Springer; 2011. p. 415-513.

4. de Winter JP, de Vries MA, Zimmermann LJ. Clinical practice: noninvasive respiratory support in newborns. Eur J Pediatr 2010; 169: 777-82.

5. Patel B, Bingham R. Laryngeal mask airway and other supraglottic airway devices in paediatric practice. Continuing Education in Anaesthesia, Critical Care \& Pain 2009; 9: 6-9. 